\title{
Predictors for Conversion to Open Appendectomy in Patients Undergoing Laparoscopic Appendectomy Based on Clinical Presentations on Ultrasonography Findings and Tzanaki's Scoring
}

\author{
Vaibhav Srivastav ${ }^{1}$, Nandan Rai ${ }^{2}$, Kajal Mishra ${ }^{3}$
}

\begin{abstract}
Background: Laparoscopic appendectomy (LA) may need to be converted to open appendectomy (OA) if intraoperative complications or severity of the disease hinders with a safe laparoscopic intervention. This may be in the form of abnormal position of appendix, adhesion due to previous inflammations, appendix mass, abscess, perforated appendix and diffuse peritonitis or other pelvic or right iliac fossa pathologies or technical problems, and lack of space for dissection. Even though these pathologies can be dealt with minimal access surgery, conversion to open surgery may become mandatory in a small number of cases. The presence of comorbidities is the independent factor related to conversion during laparoscopic appendicectomy. ${ }^{1}$

Materials and methods: The study was carried out in PG Department of Surgery, SRN Hospital associated with MLN Medical College, Prayagraj from September 2018 to September 2019 after approval from the ethical committee and after obtaining written and informed consent either from patient or their legal heir. The study was conducted on the patients admitted in the Department of Surgery, SRN Hospital, MLN. Medical College between September 2018 and September 2019 who underwent conversion appendicectomy. Patients were evaluated and their complete biodata were recorded after taking detailed history. Based on history, clinical examination, laboratory investigations, and ultrasound of abdomen and pelvis, appendicitis diagnosed. The parameters studied include age, sex, previous history of acute appendicitis any lower abdominal surgeries in the past, symptoms, duration of symptoms, sign, white blood cell (WBC) count, ultrasound abdomen and pelvis findings, American Society of Anesthesiologists (ASA) grading, and intraoperative findings including reasons for conversion.

Results: Multivariable analysis incorporating these factors available to the surgeon preoperatively identified advanced age, ASA score $>2$ points, severity of adhesion in ultrasonography (USG), significantly associated with conversion. These results highlight the complex nature of the decision to convert, in as much as baseline patient characteristics, disease severity, and surgeon factor each independently impact the probability of the successful laparoscopic procedure. Conversion in our study was significantly associated with comorbidities as out of 11 patients with comorbidities [6 hypertension (HTN), 4 diabetes mellitus (DM), 1 asthma], 10 (90.90\%) were converted to OA with significant $p$ value $(p=0.00001)$. Among nine patients with ASA grade $>2$ points, eight were converted to OA. Total leukocyte count was $>12,000$ in 25 patients (41.67\%) out of which 9 patients (36\%) were converted to OA. In this study, 21 patients (35\%) had score $\leq 9$, while 39 patients $(65 \%)$ had score $\geq 10$. Eleven patients $(52.38 \%$ ) were converted to OA out of 21 having score $\leq 9$ in comparison to 1 patient (2.56\%) out of 39 patients having score $\geq 10$.

Conclusion: We identified preoperatively, predictors for conversion of LA to OA consisting of age $\geq 40$, comorbidity, ASA grade $>2$ point, leukocytosis, right iliac fossa lump and Tzanaki's score $<9$ point. By using this, we proceed directly to OA under these circumstances may reduce operative time and expenses by conversion to OA.

Keywords: Appendectomy, Conversion, Predictors, Tzanaki's score.

World Journal of Laparoscopic Surgery (2020): 10.5005/jp-journals-10033-1401
\end{abstract}

\section{INTRODUCTION}

Acute appendicitis is one of the most common differential diagnoses of acute abdomen and a common intra-abdominal condition requiring emergency surgery. ${ }^{2}$ It can either be managed conservatively using Ochsner-Sherren regimen or operatively by laparoscopic/open appendectomy (OA).

Complicated appendicitis includes obese, older patients, and pregnant women.

In this era of advance technology and minimal access surgery, laparoscopic appendicectomy has gained much popularity owing to its suggested advantages like less postoperative pain, faster recovery, lower wound infection rates shorter hospital stay, and higher cosmetic satisfaction. ${ }^{3}$ Laparoscopic appendicectomy may need to be converted to open appendicectomy if intraoperative complications or severity of the disease hinders with a safe
${ }^{1-3}$ Department of General Surgery, Moti Lal Nehru Medical College, Allahabad, Uttar Pradesh, India

Corresponding Author: Nandan Rai, Department of General Surgery, Moti Lal Nehru Medical College, Allahabad, Uttar Pradesh, India, Phone: +91 8887743234, e-mail: nandanrai4@gmail.com

How to cite this article: Srivastav V, Rai N, Mishra K. Predictors for Conversion to Open Appendectomy in Patients Undergoing Laparoscopic Appendectomy Based on Clinical Presentations on Ultrasonography Findings and Tzanaki's Scoring. World J Lap Surg 2020;13(2):74-76.

Source of support: Nil

Conflict of interest: None

laparoscopic intervention. This may be in the form of abnormal position of appendix, adhesion due to previous inflammations, 
appendix mass, abscess, perforated appendix and diffuse peritonitis or other pelvic or right iliac fossa pathologies or technical problems, and lack of space for dissection. Even though these pathologies can be dealt with minimal access surgery, conversion to open surgery may become mandatory in a small number of cases. The presence of comorbidities is the independent factor related to conversion during laparoscopic appendicectomy. ${ }^{1}$

Conversion from laparoscopic to open appendicectomy known as conversion appendicectomy (CA), further increases the operative time, along with loss of benefits of minimal access surgery. Therefore, developing a preoperative criterion to decide ideal operative approach for individual may be useful.

\section{Materials and Methods}

The proposed study titled "Predictors for conversion to OA in patients undergoing laparoscopic appendectomy (LA) based on clinical presentations, ultrasonography (USG) findings and Tzanaki's scoring" was carried out in PG Department of Surgery, SRN Hospital associated with MLN Medical College, Prayagraj from September 2018 to September 2019 after approval from the ethical committee and after obtaining written and informed consent either from patient or their legal heir.

The study was conducted on the patients admitted in the Department of Surgery, SRN Hospital, MLN Medical College between September 2018 and September 2019 who underwent conversion appendectomy.

Patients were evaluated and their complete biodata were recorded after taking detailed history. Based on history, clinical examination, laboratory investigations, and ultrasound of abdomen and pelvis, appendicitis diagnosed. The parameters studied include age, sex, previous history of acute appendicitis any lower abdominal surgeries in the past, symptoms, duration of symptoms, sign, white blood cell (WBC) count, ultrasound abdomen and pelvis findings, American Society of Anesthesiologists (ASA) grading, and intraoperative findings including reasons for conversion.

\section{Results}

In our study of 60 patients undergoing appendicectomy, conversion to OA occurred in 12 patients (20\%). The conversion rate in this study much higher than other studies performed before because of small sample size of this study.

In our study, age group of 10-39 years had lower rate of conversion in comparison to age group of $\geq 40$ years. Out of 60 patients, 12 patients converted to open out of which 8 patients (66.67\%) were $\geq 40$ years, with significant $p$ value ( $p=0.000124)$. A study conducted by Manuneethimaran et al. ${ }^{4}$ shows almost similar age group $(48 \pm 16)$ associated with more conversion with significant $p(<0.01)$ value. Other study by Antonacci et al. ${ }^{5}$ had similar age group $(36 \pm 19.3)$ associated with more conversion with significant $p(<0.001)$ value.

In our study, 38 patients (63.33\%) were male, while 22 patients (36.67\%) were female, out of which male patients had more conversion (21.05\%) in comparison to female patients (18.18\%) which did not have significant association ( $p=0.946606)$.

Conversion in patients with comorbid conditions in our study had significant association as out of 11 patients with comorbid conditions [6 hypertension (HTN), 4 diabetes mellitus (DM), 1 asthma], 10 (90.90\%) were converted to OA with significant $p$ value ( $p=0.00001)$ in Fisher's exact test. A similar study was performed by Antonacci et al. ${ }^{5}$ in which conversion rate was high with significant $p$ value $(p=0.001)$. Another study conducted by Suresh Kumar et al. ${ }^{6}$ also has similar conversion in diabetic patient as 3 underwent conversion out of 12 patients of DM and $16 \%$ conversion in hypertensive patients.

Another predictor for conversion to OA in our study is ASA grade $>2$ points. Out of 60 patients, there were 51 patients (85\%) with ASA grade 1 point, while 9 (15\%) patients were ASA grade $>2$ point. Among nine patients, eight were converted to OA with ASA grade $>2$ point. There was a significant association with conversion of ASA grade to open procedure $(p=0.001)$. A similar study conducted by Wagner et al. ${ }^{7}$ in which 12 patients (29.7\%) out of 39 patients were converted to OA with ASA grade $>2$ points, which is significant with $p=0.001$.

In our study, leukocyte count was $>12,000$ in 25 patients (41.67\%) out of which 9 patients (36\%) were converted to OA with significant $p$ value ( $p=0.021947)$ in comparison to normal leukocyte count. Study conducted by Manuneethimaran et al. ${ }^{4}$ had the similar result the high WBC count is associated with higher conversion to open procedure with WBC count $(16,380 \pm 1,015)$ having significant $p$ value $(p \leq 0.01)$

Tzanaki's score is a diagnostic score which includes right iliac fossa tenderness, rebound tenderness, leukocytosis $(>12,000)$, and USG finding. In this study, 21 patients (35\%) had score $\leq 9$, while 39 patients $(65 \%)$ had score $\geq 10$. There was 11 patients $(52.38 \%)$ converted to OA out of 21 having score $\leq 9$ in comparison to 1 patient (2.56\%) having score $\geq 10$. This shows significant association Tzanaki's score to more conversion in population having $\leq 9$ score while less conversion in population having $\geq 10$ score.

In our study, USG finding was included in Tzanaki's scoring system, so individual finding of USG is not significantly associated with conversion to open appendicectomy. In general, USG showing as appendicular mass, appendicular adhesion, pericecal adhesion, and perforation had higher impact on decision on conversion. Study by Suresh Kumar et al. ${ }^{6}$ has similar result with association of USG finding.

Other clinical features, such as pain, fever, nausea, and vomiting, were not significantly associated with conversion in our study as statically insignificant value ( $p \geq 0.05$ ). Manuneethimaran et al. ${ }^{4}$ study having similar result to pain, fever, nausea, and vomiting is not a predictor with insignificant $p$ value $(p \geq 0.05)$ for conversion of LA to OA.

\section{Discussion}

In our study, by univariable analysis, a number of risk factors for conversion to OA were identified. These include advanced age, male sex, ASA score $>2$ points, higher leukocyte count, and severity of inflammation as shown in USG (pericecal adhesion, periappendicular adhesion, perforated appendix with peritonitis, gangrenous appendix).

Multivariable analysis incorporating these factors available to the surgeon preoperatively identified advanced age, ASA score $>2$ points, and severity of adhesion in USG are significantly associated with conversion. These results highlight the complex nature of the decision to convert, in as much as baseline patient characteristics, disease severity, and surgeon factors, as each independently impacts the probability of successful laparoscopic procedure. Thus, a careful assessment of the patient risk factors, blood investigations, and Tzanaki's scoring will help segregate patients into one group which can be safely taken up for laparoscopic appendectomies without much risk of conversion to OA and 
another group that is predicted to have significant conversion rates, hence performing open appendectomies will bear better consequences.

\section{CONCLUSION}

We identified preoperatively, predictors for conversion of LA to OA consisting of age $\geq 40$, comorbidities, ASA grade $>2$ points, leukocytosis, right iliac fossa lump and Tzanaki's score $<9$ points. By using this, we proceed directly to OA under the circumstances predicting higher risk for conversion of laparoscopic to open appendectomies, thereby reducing the cost, effort, and ensuring better patient outcome.

\section{References}

1. Ricci C, Taffurelli G, Monari F, et al. Laparoscopic appendectomy: which factors are predictors of conversion? A high-volume prospective cohort study. Int J Surg 2015;21:103-107. DOI: 10.1016/ j.ijsu.2015.06.089.
2. Addiss DG, Shaffer N, Fowler BS, et al. The epidemiology appendicitis and appendectomy in the United States. Am J Epidemiol 1990;132(5):910-925. DOI: 10.1093/oxfordjournals.aje.a115734.

3. Garg CP, Vaidya BB, Chengalath MM. Efficacy of laparoscopy in complicated appendicitis. Int J Surg 2009;7(3):250-252. DOI: 10.1016/ j.ijsu.2009.04.007.

4. Manuneethimaran T, Mohanapriya T, Arun Kumar K, et al. The risk factors for conversion of laparoscopic appendicectomy to open appendicectomy. Dent Med Sci 2015;14(10 Ver. X):33-36.

5. Antonacci N, Ricci C, Taffurelli G, et al. Laparoscopic appendectomy: which factors are predictors of conversion? A high-volume prospective cohort study. Int J Surg 2015;21:103-107. DOI: 10.1016/ j.ijsu.2015.06.089.

6. Suresh Kumar N, Jai Vinod Kumar R. Factors affecting conversion of laparoscopic appendectomy to open surgery in a tertiary hospital in South India: a prospective study. J Surg 2016;2(2):1-4. DOI: 10.1055/s0036-1583205.

7. Wagner PL, Eachempati SR, Aronova A, et al. Contemporary predictors of conversion from laparoscopic to open appendectomy. Surg Infect (Larchmt) 2011;12(4):261-266. DOI: 10.1089/sur.2010.079. 